\title{
Development in the Etiologic Diagnosis of Chagas Disease

\author{
Mario E Camargo
}

Rua Pombal 133, 01253-010 São Paulo, SP, Brasil

Key words: Chagas disease - etiologic diagnosis

The etiologic diagnosis of Chagas disease has presented up to now a continuous and progressive development, with more and more precise, sensitive and practical procedures. Since when Carlos Chagas described Trypanosoma cruzi, and next its role as the agent of the disease he also discovered and carries his name, a large amount of research has been done with the purpose of ensuring its diagnosis.

In this meeting we will have the opportunity and the pleasure of hearing from experts, and changing experiences, on the present aspects of this diagnosis, either parasitological, biomolecular or serological. Complementing coordination on the parasitological diagnosis in this round-table, by $\mathrm{Dr}$ Alejandro Luquetti, I should like to introduce the presentation on serodiagnosis by Professor Eufrosina Setsu Umezawa, and following discussions, by summarizing the main aspects of the work on serodiagnosis which have been developed at the Instituto de Medicina Tropical de São Paulo.

Chagas disease serodiagnosis, which started in 1913 with the complement fixation test described by Guerreiro and Machado, still presents many problems, a few pertinent to serodiagnosis in general, as interferring signals and cut-off selection, others directly related to parasite antigens, as well as to the patients' humoral responses. The high antigenic complexity of $T$. cruzi and its variability in evolutive forms and strains, as well as antigenic communities with related parasites represent difficulties in the way of standardizing a universal, reference test, for serodiagnosis. Antibody response at the same time presents marked differences along the phases of the disease, adding more complexity to the problem.

For thirty years or more, at the Serology and Seroepidemiology Laboratory of the Instituto, Chagas disease serology has been a constant study

Fax: +55-11-287-2428

Rceived 9 June 1999

Accepted 9 August 1999 subject. Collaboration of colleagues as Sumie Hoshino Shimizu, Antonio Walter Ferreira, Eufrosina Setsu Umezawa, Anna Maria Simonsen Stolf, Maria Carolina Soares Guimarães, Benedito Anselmo Peres, José Roberto Mineo, Katia Primavera, Tereza Keiko N Sugahara, and myself, plus staff members of other brazilian and foreign Institutions in North, Central and South America, allowed for the development and evaluation of new tests, as immunofluorescence, immunoenzymatic, hemagglutination, flocculation and immunoblot assays, the study of antigens, cross-reactions, tests standardizations, as well as the production of stable and practical reagents. In several developments the Laboratory was pioneer, as of a practical immunofluorescence assay; in removing rheumatoid factor interferences in IgM assays; in developing a stable, liquid reagent for hemagglutination; in the development of Elisa and Immunoblot assays with T. cruzi excretion and secretion antigens from trypomastigotes in cell cultures (TESA). These assays were shown as very sensitive for IgG and IgM antibodies and furnishing characteristic reactivity patterns for acute and chronic Chagas disease, while immunoblot negative for Leishmania antibodies; in developing a Universal Elisa for Chagas Disease Serodiagnosis, with an association of T. cruzi recombinant antigens. Produced by Prof. Franco da Silveira (Molecular Biology, Parasitology, Escola Paulista de Medicina, São Paulo, Brazil) and by a few foreign centers (CYTED-D), these recombinant peptides were selected in our Laboratory according to sensitivity and specificity with a large number of serum samples from patients of different endemic areas in Brazil and in several countries in South and Central America. A careful study was then performed in our Laboratory, with these recombinants after isotope-labeling, so as to determine and overcome their competition on coating the plates for Elisa.

In the development of the different tests, association with epidemiological and clinical researchers has been of paramount importance, specially with Professors J Rodrigues Coura and MA Shikanai Yasuda.

In collaboration with the Brazilian Ministry of Health, seroprevalence of Chagas disease was es- 
tablished in a survey covering the country, by sampling the population of every municipality in all states, with the exception of the State of São Paulo and the Federal District. For this purpose, the Laboratory produced all the reagents and performed a continuous test-control of the 14 collaborating laboratories throughout Brazil, in a work that took about four years. Associated with data from a parallel triatomine survey, seroprevalence results have been an indicator for Public Health measures against Chagas disease natural transmission in Brazil. It has been taken as a model for similar surveys in other countries where Chagas disease is also present. 